\title{
Formation of Supermassive Black Holes through Fragmentation of Torodial Supermassive Stars
}

\author{
Burkhard Zink, ${ }^{1}$ Nikolaos Stergioulas, ${ }^{2}$ Ian Hawke, ${ }^{3,4}$ Christian D. Ott, ${ }^{4}$ Erik Schnetter, ${ }^{5,4}$ and Ewald Müller ${ }^{1}$ \\ ${ }^{1}$ Max-Planck-Institut für Astrophysik, Karl Schwarzschild-Strasse 1, 85741 Garching bei München, Germany \\ ${ }^{2}$ Department of Physics, Aristotle University of Thessaloniki, Thessaloniki 54124, Greece \\ ${ }^{3}$ School of Mathematics, University of Southampton, Southampton SO17 1BJ, United Kingdom \\ ${ }^{4}$ Max Planck Institut für Gravitationsphysik, Albert Einstein Institut, 14476 Golm, Germany \\ ${ }^{5}$ Center for Computation and Technology, Johnston Hall, Baton Rouge, Louisiana 70803, USA
}

(Received 11 February 2005; published 26 April 2006)

\begin{abstract}
We investigate new paths to supermassive black hole formation by considering the general relativistic evolution of a differentially rotating polytrope with a toroidal shape. We find that this polytrope is unstable to nonaxisymmetric modes, which leads to a fragmentation into self-gravitating, collapsing components. In the case of one such fragment, we apply a simplified adaptive mesh refinement technique to follow the evolution to the formation of an apparent horizon centered on the fragment. This is the first study of the onset of nonaxisymmetric dynamical instabilities of supermassive stars in full general relativity.
\end{abstract}

DOI: 10.1103/PhysRevLett.96.161101

PACS numbers: 04.40.Dg, 04.25.Dm, 04.70.Bw

The formation of black holes from neutron stars, iron cores, or supermassive stars is expected to be associated with a characteristic gravitational wave signal which may give information about the collapse dynamics and the physical environment of such objects. Therefore, and given that gravitational wave detectors are already taking data or are coming online, it is of prime importance to understand the dynamical features of the gravitational collapse of hydrodynamical systems.

The prototypical model of stellar collapse is an equilibrium polytrope subject to a radial or quasiradial perturbation growing on a dynamical time scale. In spherical symmetry, every general relativistic polytrope with index $N=3$ is unstable to radial oscillations [1] - in turn, there exists a critical $N_{\mathrm{c}}<3$ for which the star is marginally stable. Without spherical symmetry, rotation can increase this critical value again [2]. The black hole formation from the collapse of uniformly and differentially rotating polytropes induced by this instability is a well-investigated phenomenon, either with restriction to axisymmetry [3-9] or without [10-13]. In the gauge choices usually employed, the dynamical behavior of the system shows a radial contraction of the star, accompanied by the formation of an apparent horizon at late times.

Black hole formation from a dynamically unstable nonaxisymmetric mode, however, has not been modeled so far. Possible scenarios range from the development of a bar mode, subsequent transport of angular momentum into the shell and collapse of the central object, to fragmentation and off-center production of one or several black holes. In Newtonian theory, instabilities and fragmentation have received considerable attention, specifically in the context of binary formation from protostellar disks (e.g., [14-18] and references therein) and compact object production in stellar core collapse (e.g., [19-21] and references therein). In [11], the authors also report signatures of an $m=4$ fragmentation behavior in the collapse and centrifugal bounce of an $N=1$ polytrope, but could not determine the final state due to resolution issues.

The cooling evolution of supermassive stars can be approximately described by the $N=3$ mass-shedding sequence when the angular momentum transport time scales are short compared to the cooling time scale [22], so that uniform rotation is enforced. This sequence has a turning point for the onset of a quasiradial instability, and numerical experiments confirm that the collapse remains axisymmetric [23]. If the star is differentially rotating, the cooling sequence is less constrained and might end in a transition to nonaxisymmetric instability [24-26]. The canonical expectation that a supermassive star produces one central black hole with a low-mass accretion disk might thus not be appropriate for differentially rotating configurations.

In this Letter, we consider the production of a black hole through the fragmentation of a general relativistic polytrope. We focus on $N=3$ polytropes to model the radiation-pressure dominated equation of state of a supermassive star. The softness of the equation of state enhances the instability of the fragments compared to the common choice $N=1$ for neutron stars. To represent this process accurately on a grid, we make use of an adaptive mesh refinement technique, since a possibly highly deformed apparent horizon needs to be located in some region of the domain which is unknown in advance.

The investigation of the collapse of differentially rotating supermassive stars by Saijo [27] was based on a sequence of relativistic $N=3$ polytropes with a parameterized rotation law of the commonly used form $j(\Omega)=$ $A^{2}\left(\Omega_{\mathrm{c}}-\Omega\right)$, where $\Omega_{\mathrm{c}}$ is the angular velocity at the center, and the parameter $A$ specifies the degree of differential rotation $(A \rightarrow \infty$ is uniform rotation). The sequence was constrained by a constant central density $\rho_{\mathrm{c}}=3.38 \times$ $10^{-6}$ in units $K=G=c=1$, and the choice $A / r_{\mathrm{e}}=1 / 3$, where $r_{\mathrm{e}}$ denotes the equatorial coordinate radius. 
To examine the indirect collapse by fragmentation of a polytrope with toroidal shape, we choose a model with the same central density as in Saijo's [27] models, but with a ratio of polar to equatorial coordinate radius $r_{\mathrm{p}} / r_{\mathrm{e}}=0.24$. The ratio of rotational kinetic energy to gravitational binding energy is $T /|W|=0.227$. While the critical limit for the dynamical $f$-mode instability in uniform density, uniformly rotating Maclaurin spheroids is $(T /|W|)_{\text {dyn }}=0.2738$ (e.g., [28]), recent investigations of the stability of soft $(N \sim 3)$ differentially rotating polytropes in Newtonian gravity [29-32] have shown that the Maclaurin approximation is inappropriate for such systems, and generally find the critical $(T /|W|)_{\text {dyn }}$ to be below the Maclaurin value. Consequently, the toroidlike star considered in this study might be unstable to nonaxisymmetric perturbations. Here we present the first investigation of black hole formation through this instability.

All simulations have been performed in full general relativity. The only assumption on symmetry is a reflection invariance with respect to the equatorial plane of the star. The gauge freedom is fixed by the generalized $1+\log$ slicing condition for the lapse function [33] with $f(\alpha)=$ $2 / \alpha$, and by the hyperbolic-type condition suggested in [8] for the shift vector.

The computational framework is the CACTUS, which also provides a module to solve the geometric part of the field equations in the well-known BSSN form [34-36]. In addition, the CARPET driver [37] is used for mesh refinement in CACTUS. The hydrodynamics part of the field equations is evolved using the high-resolution shock-capturing piecewise parabolic method-Marquina implementation in the WHISKY module [12,38], and a gamma law equation of state $(P=\rho \epsilon / N)$. We are thus using a set of well-tested tools to evolve the general relativistic hydrodynamics equations.

To numerically construct the axisymmetric initial model described in the introduction, we use the $R N S$ initial data solver [39]. With the parameters described above, the model has toroidlike structure, with an off-center density maximum, but a nonzero central density. After mapping the model to the hierarchy of Cartesian grids provided by CARPET, a small perturbation of the form

$$
\rho(x) \rightarrow \rho(x)\left[1+\frac{1}{\lambda r_{\mathrm{e}}} \sum_{m=1}^{4} \lambda_{\mathrm{m}} B \varpi \sin (m \phi)\right]
$$

is applied with $\lambda_{\mathrm{m}}=0,1$ and $\lambda=\sum_{\mathrm{i}} \lambda_{\mathrm{i}}$. Here, $\varpi$ denotes the cylindrical coordinate radius. In addition, the polytropic constant $K$ is reduced by $0.1 \%$ to induce collapse if the model is radially unstable. After perturbing the model, the constraint equations are not solved again, since the amplitude $B$ is chosen such that the violation of the constraints by the initial perturbation is about an order of magnitude smaller than that caused by the systematic error induced by the $m=4$ symmetry of the Cartesian grid (cf. Figs. 1 and 2). A typical amplitude used is $B=10^{-3}$, but a

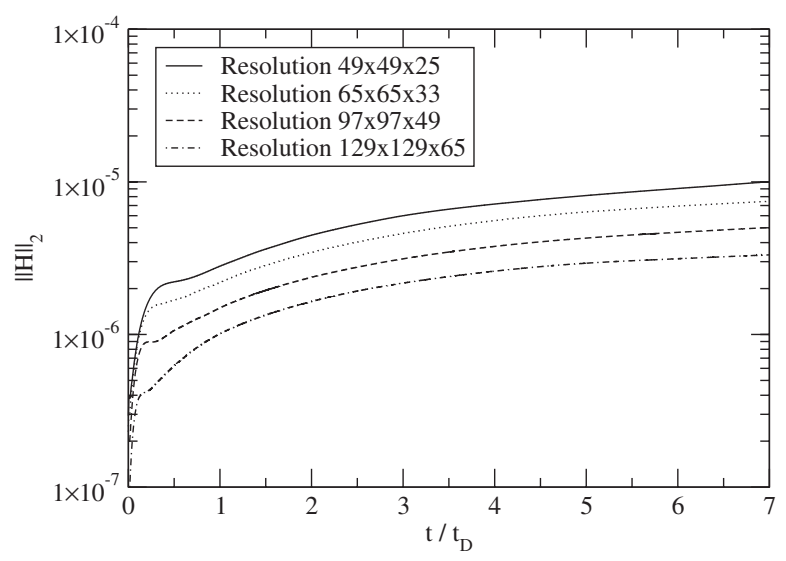

FIG. 1. Time evolution of the $L^{2}$ norm of the Hamiltonian constraint for different resolutions. The time is normalized to the dynamical time scale $t_{\mathrm{D}}=r_{\mathrm{e}} \sqrt{r_{\mathrm{e}} / M}$.

test with $B=10^{-4}$ has also been performed to ensure that the system is not sensitive to the choice of $B$ [40].

For most simulations, a fixed box-in-box mesh refinement with 5 levels is used to accurately resolve the central high-density ring. The three innermost grids cover the star, while the two outermost ones push the outer boundaries to $6.4 r_{\mathrm{e}}$. The typical resolution used was $65 \times 65 \times 33$ per grid patch, leading to a central resolution of $\delta_{\mathrm{x}} \approx 10^{-2} r_{\mathrm{e}}$. However, simulations with $49 \times 49 \times 25,97 \times 97 \times 49$, and $129 \times 129 \times 65$ points per grid patch were also performed to demonstrate decreasing constraint violation with increasing resolution; for the last setup, a simulation with a uniform grid setup would need to cover the equatorial plane of the star alone with 320 grid points to achieve the same central resolution.

To determine the amplitude of a specific mode in the equatorial plane, we perform a projection onto Fourier modes at certain coordinate radii $[41,42]$. Care must be taken in interpreting the results when the system deviates

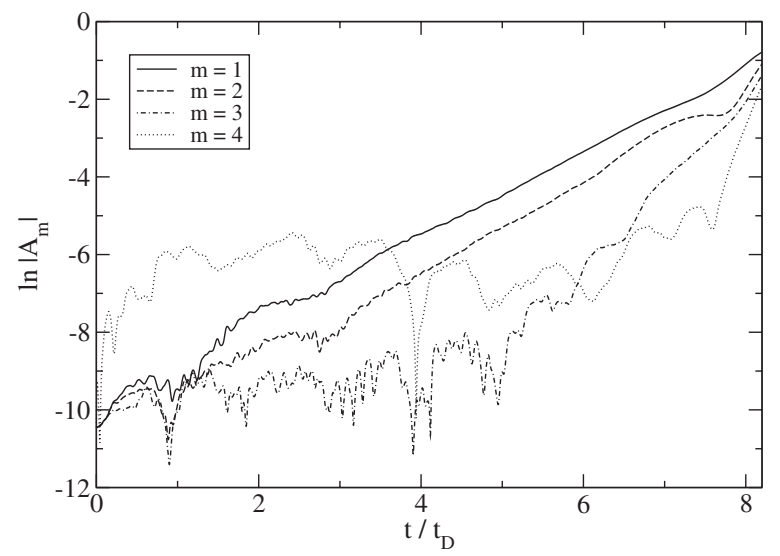

FIG. 2. Time evolution of the mode amplitudes in the standard resolution $(65 \times 65 \times 33$ points per patch). The amplitudes are obtained from a Fourier decomposition of the density profile on the equatorial plane circle at $\varpi=0.25 r_{\mathrm{e}}$, the initial radius of highest density. 
significantly from axisymmetry, since the interpretation of the projection curve as a circle assumes $\partial_{\phi}$ to be a Killing vector. In addition to the Fourier extraction, we monitor the evolution of the rest mass and the constraints.

For the standard grid setup $(65 \times 65 \times 33$ points per patch), and with $\lambda_{1 \ldots 4}=1$, the evolution of the moduli of the equatorial Fourier components at the initial radius of highest density is shown in Fig. 2. It is evident that, initially, the $m=4$ component induced by the Cartesian grid is dominant. However, the star is unstable to $m=1$ and $m=2$, and these modes consequently grow into the nonlinear regime, their $e$-folding times being rather close.

The rest mass is conserved numerically within $1.8 \%$ (with $65 \times 65 \times 33$ points per patch) to within $0.2 \%$ $(129 \times 129 \times 65$ points per patch). An approximate measurement of the $e$-folding times and mode frequencies can be obtained within an error of 5\%-10\% related to ambiguities in defining the interval of extraction. All setups show consistent results within this uncertainty. In units of the dynamical time scale, which is defined here as $t_{\mathrm{D}}=$ $r_{\mathrm{e}} \sqrt{r_{\mathrm{e}} / M}$, the $e$-folding times are $\approx 0.93 t_{\mathrm{D}}$ for $m=1$, and $\approx 0.84 t_{\mathrm{D}}$ for $m=2$, respectively. Mode frequencies are $\approx 3.05 / t_{\mathrm{D}}$ for $m=1$ and $\approx 3.31 / t_{\mathrm{D}}$ for $m=2$, respectively.

To establish whether a black hole is formed by a fragment it is necessary to cover the fragment with significantly more resolution than affordable by fixed mesh refinement. Hence we have implemented a simplified adaptive mesh refinement scheme to follow the system to black hole formation: In this scheme, a tracking function, here provided by the location of a density maximum, is used to construct a locally fixed hierarchy of grids moving with the fragment. Additional refinement levels are switched on during contraction, until an apparent horizon is found.

Since the $e$-folding times for $m=1$ and $m=2$ turn out to be close, the number and interaction behavior of the fragments in the nonlinear regime depend sensitively on the initial perturbation. Thus setups with $\lambda_{m}=\delta_{1 m}$ and $\lambda_{m}=\delta_{2 m}$ are used to follow the formation and evolution of a specific number of fragments.

The time evolution of the equatorial plane density for $\lambda_{m}=\delta_{1 m}$ is shown in Fig. 3. While the initial model is axisymmetric, it has already developed a strong $m=1$ type deviation from axisymmetry at $t=6.43 t_{\mathrm{D}}$, which, consequently, evolves into a collapsing off-center fragment. At $t=7.45 t_{\mathrm{D}}$, we find an apparent horizon (AH), using the numerical code described in [43]. The AH is centered on the collapsing fragment at a coordinate radius of $r_{\mathrm{AH}} \approx 0.16 r_{\mathrm{e}}$, and has an irreducible mass of $M_{\mathrm{AH}} \approx$ $0.24 M_{\text {star }}$. Its coordinate representation is significantly deformed: its shape is close to ellipsoidal, with an axes ratio of $\sim 2: 1.1: 1$. The $\mathrm{AH}$ is covered by three refinement levels and 50 to 100 grid points along each axis.

The evolution using $\lambda_{m}=\delta_{2 m}$ is shown in Fig. 4. Two orbiting and collapsing fragments are forming. However, even with the adaptive mesh refinement method we use,

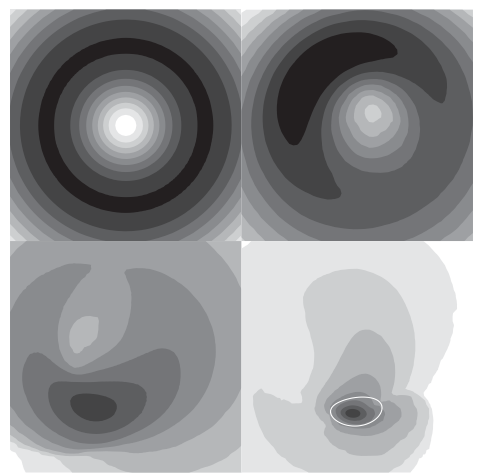

FIG. 3. Time evolution of the equatorial plane density using the perturbation parameters $\lambda_{m}=\delta_{1 m}$. Shown are isocontours of the logarithm of the rest-mass density. The four snapshots extend to $0.37 r_{\mathrm{e}}$ and are taken at $t / t_{\mathrm{D}}=0,6.43,7.14$, and 7.45, respectively. They show the formation and collapse of the fragment produced by the $m=1$ instability. The last slice contains an $\mathrm{AH}$ demarked by the thick white line. Note that the shades of gray used for illustration are adapted to the current maximal density at each time, and that darker shades denote higher densities.

constraint violations prevent us from continuing the simulation to the formation of AHs. Cell-based adaptive mesh refinement, a better choice of gauge, or methods based on discrete analysis (e.g., [44]) might be required in this case.

A preliminary gravitational wave signal is shown in Fig. 5 for a $10^{6} M_{\odot}$ star at a distance of $1 \mathrm{Gpc}$. It is only possible to derive a lower limit on the gravitational wave amplitude with the current techniques, since the last part of the evolution is outside of the causal past of most detectors. With these limitations, we get a signal amplitude of about $10^{-18}\left(M / 10^{6} M_{\odot}\right) /(d / 1 \mathrm{Gpc})$, and a frequency of about $5.5\left(10^{6} M_{\odot} / M\right) \mathrm{mHz}$.

To summarize, we have studied fragmentation and black hole formation of a general relativistic equilibrium polytrope of index $N=3$. The polytrope has been shown to be unstable to cylindrical perturbations of the form $r \sin (m \phi)$, with $m=1,2$, which consequently grow to one or more

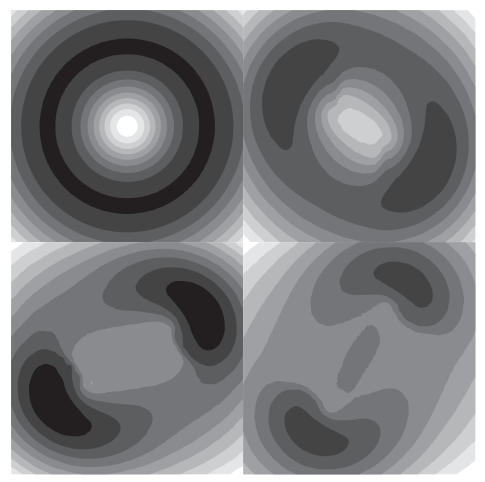

FIG. 4. Time evolution of the equatorial plane density using the perturbation parameters $\lambda_{m}=\delta_{2 m}$. The snapshot times are the same as in Fig. 3. In this case, two fragments are forming. Constraint violations have forced us to terminate the simulation before AHs could be located, (see main text). 


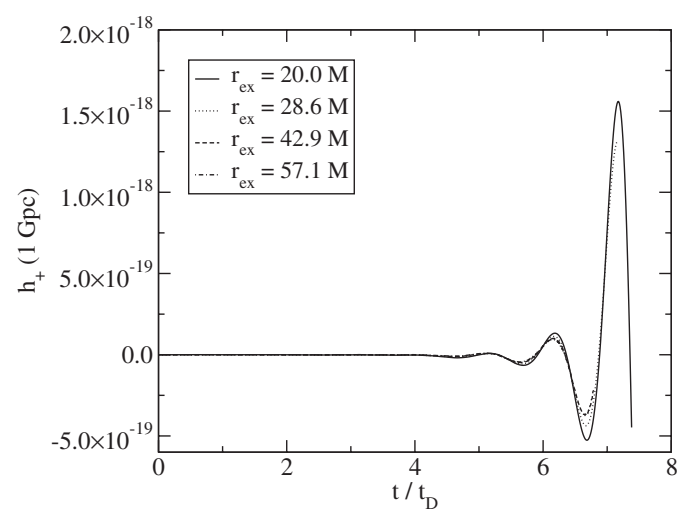

FIG. 5. Example of a gravitational wave signal. The extracted signal is normalized to a $10^{6} M_{\odot}$ source at a distance of $1 \mathrm{Gpc}$, and an observer inclination of $\theta=0$. Note that $t_{\mathrm{D}} \approx 182 \mathrm{~s}$ in this case.

self-gravitating fragments. We have applied an adaptive mesh refinement method to resolve the system accurately. In the $m=1$ case, we have found an apparent $\mathrm{AH}$ in the spacetime, indicating that a black hole has formed.

The dynamics of a nonaxisymmetric single-star collapse of this type differ significantly from the quasiradial cases usually investigated. From the often considered case of quasiradial collapse, to bar formation and subsequent collapse, to fragmentation and fragment inspiral, we have a range of possible dynamical scenarios which may be connected to discernible observable features in their gravitational wave signature. In that sense, the evolution presented here can be considered as an example of such processes.

We would like to thank J. Thornburg for his AH finder. We used CACTUS and the CACTUSEINSTEIN infrastructure, the WHISKY code developed by the European Network on Sources of Gravitational Waves, and the CARPET code for mesh refinement. In addition, we are grateful to L. Rezzolla, J. Font, A. Watts, N. Andersson, M. Saijo, M. Shibata, and A. Nagar for helpful comments. This work has been supported in part by the DFG SFB-TR7 on Gravitational Wave Astronomy. Calculations were performed on computing resources at MPA and AEI.

[1] S. Chandrasekhar, Astrophys. J. 140, 417 (1964).

[2] W. Fowler, Astrophys. J. 144, 180 (1966).

[3] T. Nakamura, Prog. Theor. Phys. 65, 1876 (1981).

[4] R. F. Stark and T. Piran, Phys. Rev. Lett. 55, 891 (1985).

[5] M. Shibata, Prog. Theor. Phys. 104, 325 (2000).

[6] M. Shibata and S. Shapiro, Astrophys. J. 572, L39 (2002).

[7] M. Shibata, Phys. Rev. D 67, 024033 (2003).

[8] M. Shibata, Astrophys. J. 595, 992 (2003).

[9] Y. I. Sekiguchi and M. Shibata, Phys. Rev. D 70, 084005 (2004).

[10] M. Shibata, T. W. Baumgarte, and S. L. Shapiro, Phys. Rev. D 61, 044012 (2000).
[11] M. D. Duez, S. L. Shapiro, and H. J. Yo, Phys. Rev. D 69, 104016 (2004).

[12] L. Baiotti, I. Hawke, P. J. Montero, F. Löffler, L. Rezzolla, N. Stergioulas, J. A. Font, and E. Seidel, Phys. Rev. D 71, 024035 (2005).

[13] L. Baiotti, I. Hawke, L. Rezzolla, and E. Schnetter, Phys. Rev. Lett. 94, 131101 (2005).

[14] R. Durisen, R. Gingold, J. Tohline, and A. Boss, Astrophys. J. 305, 281 (1986).

[15] J. Tohline and I. Hachisu, Astrophys. J. 361, 394 (1990).

[16] I. Bonnell and M. Bate, Mon. Not. R. Astron. Soc. 271, 999 (1994).

[17] B. Pickett, R. Durisen, and G. Davies, Astrophys. J. 458, 714 (1996).

[18] R. Banerjee, R. Pudritz, and L. Holmes, Mon. Not. R. Astron. Soc. 355, 248 (2004).

[19] I. Bonnell and J. Pringle, Mon. Not. R. Astron. Soc. 273, L12 (1995).

[20] M. Davies, A. King, S. Rosswog, and G. Wynn, Astrophys. J. 579, L63 (2002).

[21] M. Shibata and Y.-I. Sekiguchi, Phys. Rev. D 71, 024014 (2005).

[22] T. Baumgarte and S. Shapiro, Astrophys. J. 526, 941 (1999).

[23] M. Saijo, T. Baumgarte, S. Shapiro, and M. Shibata, Astrophys. J. 569, 349 (2002).

[24] P. Bodenheimer and J. Ostriker, Astrophys. J. 180, 159 (1973).

[25] K. New and S. Shapiro, Astrophys. J. 548, 439 (2001).

[26] K. New and S. Shapiro, Classical Quantum Gravity 18, 3965 (2001).

[27] M. Saijo, Astrophys. J. 615, 866 (2004).

[28] J. Tassoul, Theory of Rotating Stars (Princeton University, Princeton, NJ, 1978).

[29] J. Centrella, K. New, L. Lowe, and D. Brown, Astrophys. J. 550, L193 (2001).

[30] M. Shibata, S. Karino, and Y. Eriguchi, Mon. Not. R. Astron. Soc. 334, L27 (2002).

[31] M. Saijo, Astrophys. J. 595, 352 (2003).

[32] M. Shibata, S. Karino, and E. Yoshiharu, Mon. Not. R. Astron. Soc. 343, 619 (2003).

[33] C. Bona, J. Massó, E. Seidel, and J. Stela, Phys. Rev. Lett. 75, 600 (1995).

[34] T. Nakamura, K. Oohara, and Y. Kojima, Prog. Theor. Phys. Suppl. 90, 1 (1987).

[35] M.Shibata and T. Nakamura, Phys. Rev. D 52, 5428 (1995).

[36] T. W. Baumgarte and S. L. Shapiro, Phys. Rev. D 59, 024007 (1999).

[37] E. Schnetter, S. Hawley, and I. Hawke, Classical Quantum Gravity 21, 1465 (2004).

[38] L. Baiotti, I. Hawke, P. Montero, and L. Rezzolla, Mem. Soc. Astron. Ital. 1, 210 (2003).

[39] N. Stergioulas and J. Friedman, Astrophys. J. 444, 306 (1995).

[40] We have also confirmed, by comparison to a radial dependence $\varpi^{m}$, that the nonsmoothness of the perturbation function at $\varpi=0$ for $m>1$ does not affect the evolution.

[41] K.C.B. New, J. M. Centrella, and J.E. Tohline, Phys. Rev. D 62, 064019 (2000).

[42] J.De Villiers and J. Hawley, Astrophys. J. 577, 866 (2002).

[43] J. Thornburg, Classical Quantum Gravity 21, 743 (2004).

[44] M. Tiglio, L. Lehner, and D. Neilsen, Phys. Rev. D 70, 104018 (2004). 